\title{
Information entropy in solar atmospheric fields
}

\section{Intensity photospheric structures}

\author{
G. Consolini ${ }^{1}$, F. Berrilli ${ }^{2,1}$, A. Florio ${ }^{1,2}$, E. Pietropaolo ${ }^{3,1}$, and L. A. Smaldone ${ }^{4}$ \\ ${ }^{1}$ Istituto di Fisica dello Spazio Interplanetario-CNR, 00133 Roma, Italy \\ 2 Dipartimento di Fisica, Università di Roma Tor Vergata, 00133 Roma, Italy \\ 3 Dipartimento di Fisica, Università di L'Aquila, 67010 L'Aquila, Italy \\ 4 Dipartimento di Scienze Fisiche, Università di Napoli Federico II, 80126 Napoli, Italy
}

Received 30 July 2002 / Accepted 31 January 2003

\begin{abstract}
The existence of a quasi-regular pattern in solar photospheric convective fields is an open question. In the present work, this problem is quantitatively approached by means of the normalised information entropy measure $H(r)$ as introduced by Van Siclen (1997), which reports on the information content at different scales. Images were acquired at the THEMIS telescope of the European Northern Observatory by the IPM observing mode, and at the Richard B. Dunn Solar Telescope of the National Solar Observatory.

The evaluation of $H^{\prime}(r)$ in the case of photospheric intensity binarized images shows the presence of maxima which are evidence of different prominent scales in the photospheric pattern. The relative positions of these maxima defines an ordering scale $\sim 1.6 \mathrm{Mm}$ in both instantaneous and average images. This is read as the evidence of a spatio-temporal organization in the evolution of convective pattern. The emergence of an ordering scale is discussed in the framework of pattern formation in random systems and in connection with the findings of previous works.

By averaging images with time, an increase of the information content characterized by a coherence time of $\sim 1 \mathrm{~h}$ is observed in the range of scales from 5.0 Mm to $10.0 \mathrm{Mm}$.
\end{abstract}

Key words. Sun: photosphere - Sun: granulation - methods: statistical

\section{Introduction}

The solar external layers show the effects of several physical processes occurring at different scales and depths in the Sun. Among these processes a special role is played by the convection, the main kind of energy transport in the subsurface layers. In the past, different observational and analytical methods pointed out the existence of different relevant length scales related to different patterns.

The granulation is the best documented and evident photospheric intensity pattern. It is directly visible in high resolution broadband intensity or Doppler velocity images (Spruit et al. 1990). This granulation pattern consists of an assembly of convection cells, composed by a central plasma upflow surrounded by a structured network of downflows, where hydrodynamic instabilities, as recently suggested by numerical simulations, play an important role in the generation of the granulation pattern (Stein \& Nordlund 1998; Rast 1999). These granular cells are characterized by a typical horizontal extent of $\sim 1.4 \mathrm{Mm}$ (Lawrence et al. 1999), and their typical lifetime ranges from few minutes to $\sim 15 \mathrm{~min}$. However, in spite of these short

Send offprint requests to: G. Consolini, e-mail: consolini@ifsi.rm.cnr.it lifetimes, a prolonged persistence of the granulation pattern and/or of related photospheric structures has been reported in several studies (Baudin et al. 1997; Roudier et al. 1997; Hoekzema et al. 1998; Müller et al. 2001; Getling \& Brandt 2002). In particular, averaging many hours $(\sim 1 \div 8)$ of observations, Getling \& Brandt (2002) revealed quasi-regular structures of the photospheric and subphotospheric flows, that suggests the existence of a previously unknown type of selforganization resembling the so-called target patterns observed in experiments on Rayleigh-Bénard convection. However, this point of view has been recently discussed by Rast (2002), who suggested the granulation field to be consistent with a completely random and changing flow pattern, not-requiring selforganization.

A less evident pattern is associated with mesogranulation, firstly reported by November et al. (1981) on the basis of the analysis of Doppler velocity fields. The mesogranular field seems to be characterized by an intermediate range of scales $(\sim 4 \div 7 \mathrm{Mm})$ lying between granulation and supergranulation ones. As pointed out by Rieutord et al. (2000), although different approaches have been applied to the study of mesogranulation pattern, the emerging picture is foggy and controversial. The physical origin of mesogranulation is still an open 


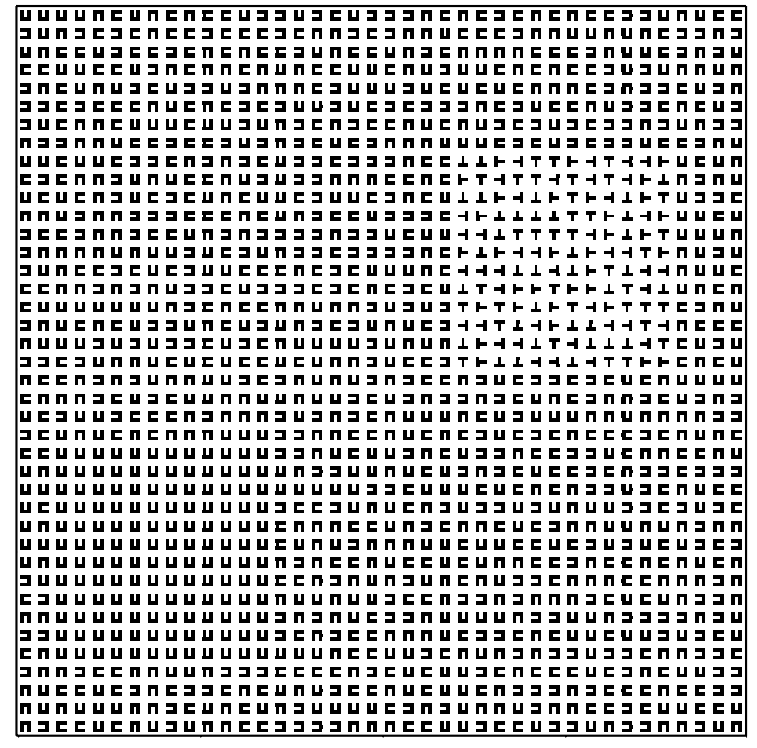

Fig. 1. An image made by a regular distribution of randomly oriented $\mathrm{U}$, and including two structures, the former consisting of $\mathrm{U}$ letters uniformly oriented, the latter of randomly oriented $\mathrm{T}$. of the two structures only one is easily visible (adapted from Legrenzi 2002).

question. Besides the traditional account of mesogranulation as cellular convection driven by superadiabaticity in the deeper layer, where neutral helium ionizes, some authors state a subsurface convective nature of mesogranular pattern (Ploner et al. 2000). Conversely, Rieutord et al. (2000) argued that mesogranulation is due to a combination of strong positive divergences, induced by highly energetic granules, and averaging effects of image analysis. Simulations by Cattaneo et al. (2001) support the idea that mesogranules owe their origin to collective interactions between the granules. Moreover, Lawrence et al. (1999) underlined the relevance of a turbulent component in the horizontal flows at the same mesogranular scales.

The recognition of quasi-regular topologies in natural patterns may be approached qualitatively by eyes and/or quantitatively by numerical methods. However, the study of natural patterns requires the definition of non-subjective quantities that may help to characterize the emergent regularities. In order to clarify the above concepts let us refer to the pattern reported in Fig. 1. Although at a first look only one ordered structure is evident, the pattern also contains a second ordered structure. As a matter of fact, this pattern consists of randomly oriented U letters plus two square structures made of T letters randomly oriented (top right region) and of U letters with the same orientation (bottom left region). This example clearly demonstrates that the classification of patterns by eye may be misleading. Thus, non-subjective methods are required for the study of natural patterns.

In the present work, we quantitatively investigate the existence, the relevance and the nature of some scales in the photospheric fields by means of the normalised information entropy as introduced by Van Siclen (1997), focusing our attention on granulation pattern. Using this new analytical approach, which is based on Shannon's information theory, we check the possible existence of a nearly ordered topology of the granular structures in single and time-averaged photospheric intensity fields. Here, the term "topology" refers to how the granulation structures are arranged in the photospheric field.

The paper is structured as follows. In Sect. 2 we present a brief mathematical description of the information entropy approach along with the results of some numerical simulations on ordered and disordered topologies. Section 3 is completely devoted to the description of the photospheric observations and of the image reduction and processing. Section 4 will present the results of the investigation on the topology of the granular structures in both single and time-averaged photospheric intensity images. A discussion of the results is also given in this section. We conclude the work with Sect. 5 where the summary and the conclusions of our analysis are reported. Appendix A reports the results of the information entropy method on some meaningful simulated patterns.

\section{The information entropy}

The investigation and the characterization of the solar granulation pattern may benefit by statistical-mathematical methods introduced in different physical frameworks. In detail, in the field of matter physics it was clearly shown that the study of the topology and the evolution of correlated random structures may take advantage of the application of Shannon's information theory (Shannon \& Weaver 1959; Beck \& Schlögl 1993). As a matter of fact, morphology and topology information may be extracted by applying the Gibbs-Shannon entropy concept. For example, Andraud et al. $(1994,1997)$ introduced an entropic measure, named configuration entropy, as a descriptor of random morphologies. Such configuration entropy is a measure of the local fluctuations of some measurable quantity over the random system, defined as follows.

Let us consider a $L \times L$ binarized (black and white) image of some physical observable quantity, and a square sliding cell of side length $l$ containing $a=l^{2}$ pixels. If $N_{k}(a)$ is the number of cells of size $a$ containing $k$ black pixels, and $N$ is the total number of sliding cells, then we can define a set of relative frequencies $p_{k}(a)$, as estimators for probabilities, according to the following expression:

$p_{k}(a)=\frac{N_{k}(a)}{N}$

Henceforth, the configuration entropy $H^{*}(a)$ will follow according to the definition:

$H^{*}(a)=-\frac{1}{\log (a+1)} \sum_{k=0}^{a} p_{k}(a) \log p_{k}(a)$.

Such a definition is equivalent to the usual Gibbs-Shannon entropy normalised by the factor $\log (a+1)$, which plays a role similar to the "multiplicative renormalization in the theory of critical phenomena" (Andraud et al. 1997). When applied to a disordered system, this entropic measure is able to extract some useful information dealing with the typical length scale at which inhomogeneities are present and so on.

A more sensitive measure of the morphological complexity in random system was introduced by Van Siclen (1997). 
This measure was called information entropy $H(l)$ because of its ability to extract the average information content at a certain length scale $l$. Moreover, this measure is able to keep information on clustering effects, as well as on periodicity or ordering. According to the information theory, the information content of a system in a configuration $i$ is directly proportional to $-\log p_{i}$, where $p_{i}$ is the a priori probability of finding the system in the $i$ th configuration. When the information content is averaged over all the possible choices it defines the information entropy $H$. Moreover, to make such a measure more sensitive to the structures at the different scale Van Siclen introduced the normalised information entropy measure $H^{\prime}=H-H_{\mathrm{r}}$, where $H_{\mathrm{r}}$ is the expected information entropy for a completely random system and $H$ is the actual information entropy. Let us briefly resume how to evaluate such a quantity.

Consider a two-level image consisting of a collection of $N$ black pixels placed on a square grid of side length $L$, and a $m \times m$ box. Let it be $p_{i}$ the probability of finding exactly $i$ black pixels of the $N$ in a $m \times m$ box if the black pixels were perfectly distributed in the random fashion. Then,

$p_{i}(m)=\left(\begin{array}{c}m^{2} \\ i\end{array}\right)\left(\begin{array}{c}L^{2}-m^{2} \\ N-i\end{array}\right)\left(\begin{array}{c}L^{2} \\ N\end{array}\right)^{-1}$

where $i$ ranges from $\max \left(0, m^{2}-L^{2}+N\right)$ to $\min \left(N, m^{2}\right)$. From here it follows that the reference information entropy of a finite, perfectly random, system is

$H_{\mathrm{r}}(m)=-\sum_{i} p_{i}(m) \log \left(p_{i}(m)\right)$.

On the other hand, the information entropy $H(m)$ for the actual configuration is

$H(m)=-\sum_{i} P_{i}(m) \log \left(p_{i}(m)\right)$

where $P_{i}(m)$ is the actual probability of finding $i$ black pixels in any $m \times m$ box, and $p_{i}(m)$ is defined according to Eq. (3). Therefore, the normalised information entropy will be

$H^{\prime}(m)=-\sum_{i}\left(P_{i}(m)-p_{i}(m)\right) \log \left(p_{i}(m)\right)$.

On the basis of the definition of $H^{\prime}(m)$ we should expect that for a completely random distribution of a collection of $N$ black pixels $H^{\prime}(m)=0$ at each length scale $m$. In Fig. 2 we report the behaviour of $H^{\prime}$ averaged on an ensemble of several $200 \times 200$ two-level images containing approx. 4000 black pixels pseudorandomly distributed. As expected $H^{\prime}(m)$ is very close to zero over the entire analyzed range of scales. The slight deviations from zero should be read as the effect of a sporadic random aggregation of some black pixels, as well as from finite size effects. We remark that the range of scales investigated has been limited to half of the linear size of the images $(m \leq 100)$ to avoid the artifacts of this analysis at larger scales due to the decreasing significativity of the box statistics (Van Siclen 1997).

Figure 3 shows a $200 \times 200$ two-level image obtained from a pseudorandom uniform distribution of the same square structure of linear dimension $m_{\mathrm{s}}=7$ pixels around the sites of a periodic square lattice with a lattice constant $m_{\mathrm{r}}=20$ pixels.

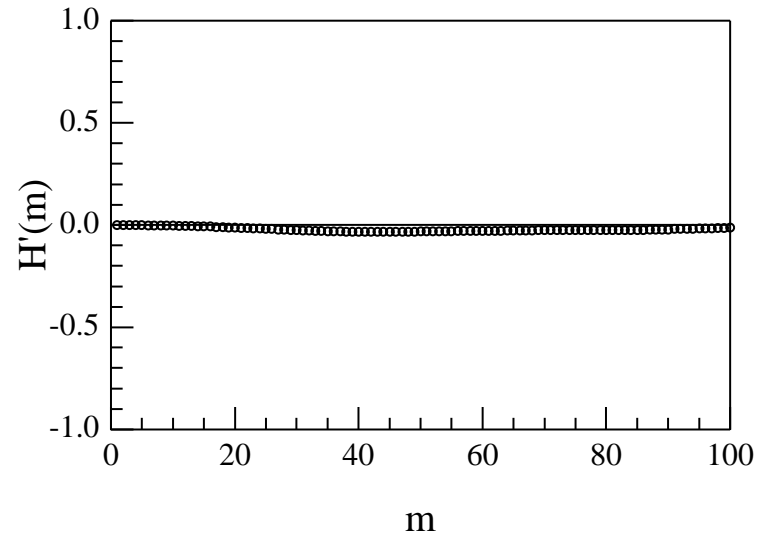

Fig. 2. $H^{\prime}(m)$ versus $m$ for a pseudorandom distribution of approx. 4000 black pixels. $H^{\prime}$ has been averaged over an ensemble of 100 different configurations.

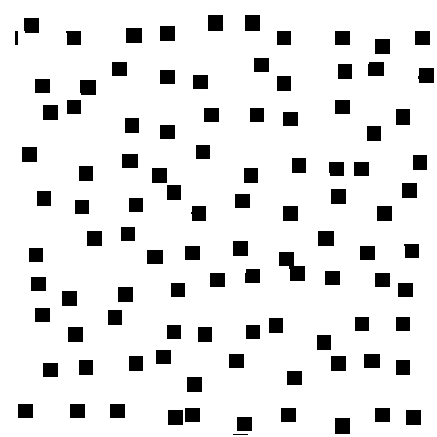

Fig. 3. A pseudorandom uniform distribution of $m_{\mathrm{s}} \times m_{\mathrm{s}}$ square structures (with $m_{\mathrm{s}}=7$ ) around the sites of a square lattice with a lattice constant $m_{\mathrm{r}}=20$ pixels. We remark that each structure is distributed inside the square cell centred on each lattice site.

The results of the $H^{\prime}$ analysis on the pattern reported in Fig. 3 are shown in Fig. 4. The behaviour of $H^{\prime}$ as a function of the sliding box dimension $m$ reveals the presence of several maxima that, according to Van Siclen (1997), can be read as follows. The first maximum, which occurs at $m_{\max } \simeq$ 12-13 pixels, is related with the lattice constant $m_{\mathrm{r}}$ and the structure dimension $m_{\mathrm{s}}$. The additional maxima reveal the existence of clusters of structures.

With reference to Fig. 4, in order to extract information on the preminent clustering scales, and to provide some insights on the ordered topology, we decompose the $H^{\prime}$ into a superposition of several contribution functions, as follows:

$H^{\prime}(m)=A_{0} m^{\alpha} \mathrm{e}^{-(m / \sigma)^{\beta}}+\sum_{k} A_{k} e^{-\left(\left(m-m_{k}\right) / \sigma_{k}\right)^{2}}$.

The first contribution on the right hand refers to the asymmetric first peak, while the second one is a superposition of Gaussian functions centred at $m_{k}$. Although the decomposition is not unique, the suggested one allows to reveal the existing hidden periodicity of the square lattice. This is particularly evident when the peak positions of the different contributions are plotted versus the odd numbers (see Fig. 5). As a matter of fact, the following relationship is found:

$m_{k}=(2 k+1) \frac{m_{\mathrm{r}}}{2}, \quad k=1,2, \ldots ., n$ 


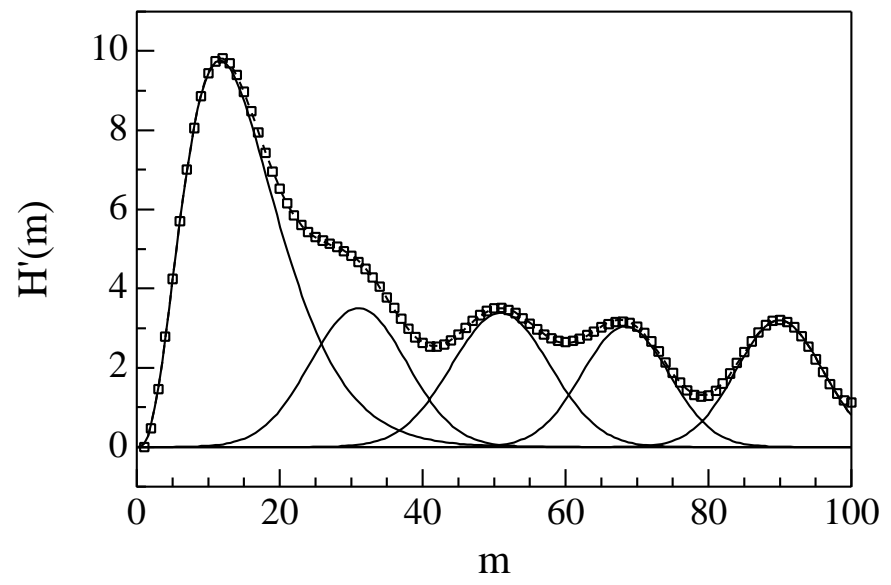

Fig. 4. The normalised information entropy $H^{\prime}$ (empty squares) relative to the pattern reported in Fig. 3. Solid lines refer to the single contributions derived by fitting $H^{\prime}$ using Eq. (7) with $k=4$.

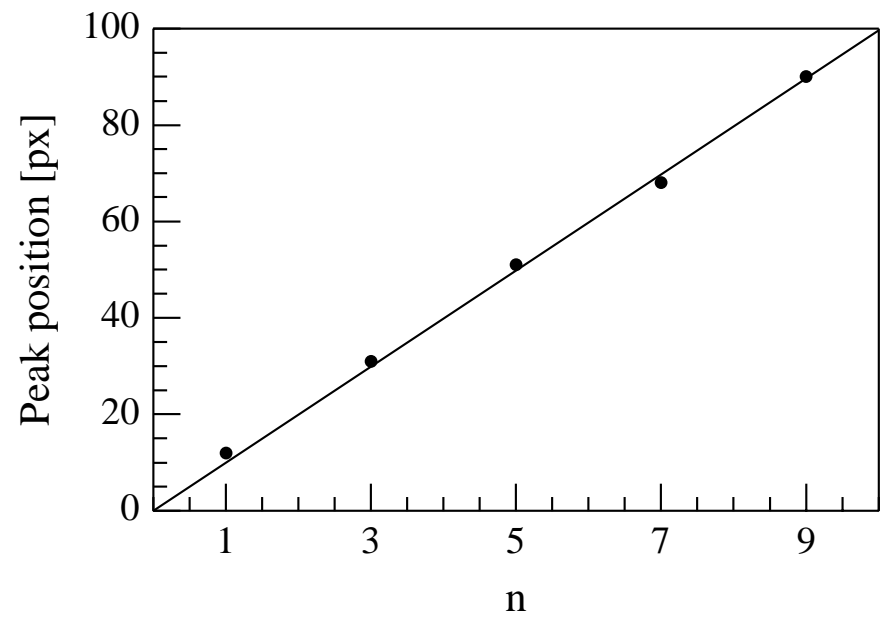

Fig. 5. The peak positions $m_{k}$ derived from the decomposition of $H^{\prime}$ plotted versus odd numbers. The solid line is a linear best fit using Eq. (7). The resulting lattice constant is $m_{\mathrm{r}}=[20.0 \pm 0.2]$.

where $m_{\mathrm{r}}$ is lattice constant. This is to say that the investigation of maxima positions can give some information on the existence of a nearly-ordered hidden lattice structure, although we cannot say anything about the real geometry of the hidden ordering. In passing through, we underline that the position of the first asymmetric peak (indicated with $k=0$ in Eq. (8)) has not been considered in the fitting procedure, being affected by the dimension of the structures and of the empty zones as discussed by Van Siclen (1997).

Before going on, let us briefly discuss and comment what is different in this kind of analysis with respect to the more usual techniques based on Fourier and/or wavelet transforms. The main difference among these methods of analysis is that the normalised information entropy $H^{\prime}$ analysis, being based on a probability measure, does not require any preliminary choice of an ad hoc set of functions or waveforms (sine-cosine waves, plane waves, wavelets, etc.) in order to decompose the field/image under investigation. Moreover, because the Fourier spectrum is related to the autocorrelation function over the field, in order to observe strong signatures of periodicities using these techniques, structures should be all of the same type (i.e. have the same shape), all oriented in the same direction, and all located in the same position inside any elementary cell. When these conditions are not fulfilled, spurious results may be found in terms of broad-band spectra (i.e. peaks may not be detectable). On the other hand, although wavelet analysis has a greater capability in finding localised isolated structures and in extracting characteristic scales, when the results are discussed in terms of spectra, this technique does not differ substantially from the Fourier transform (Lawrence et al. 2001; Starck et al. 1998). Conversely, the normalised information entropy $H^{\prime}$ analysis, looking for regularities by using a probabilistic approach, is less affected by the aforementioned aspects. In other words, while Fourier analysis looks for periodicities in the field and wavelet analysis looks for characteristic scales, the normalised information entropy $H^{\prime}$ analysis is able to extract information on the presence of statistical regularities in the mutual positions of the structures.

\section{Observations}

In order to investigate photospheric features we used two datasets of quiet granulation frames, at disk center, obtained at the THEMIS telescope of the European Northern Observatory (Tenerife) and at the Richard B. Dunn Solar Telescope (DST) of the National Solar Observatory (Sacramento Peak).

The THEMIS telescope (Observatorio del Teide, Tenerife) time series consists of broadband (5 nm $F W H M$ around $557 \mathrm{~nm})$ and monochromatic (2.1 pm $F W H M)$ images and has been acquired on July 1, 1999 (from 7:21 to 8:24 UT) with the IPM observing mode. This mode uses an Universal Birefringent Filter followed by a Fabry-Pérot interferometer (Cavallini 1998). The images were acquired with a broadband camera and, simultaneously, with a monochromatic camera at different spectral points. A more thorough description can be found in Berrilli et al. (2002). Each spectral series consisted of 19 broadband and 19 monochromatic images. The images were acquired with two different integration times, $40 \mathrm{~ms}$ and $200 \mathrm{~ms}$ for broadband and monochromatic frames, respectively. The two 12-bit CCD cameras (Berrilli et al. 1997) are binned to $256 \times 256$ pixels. The Field of View $(\mathrm{FoV})$ is $34^{\prime \prime} \times 34^{\prime \prime}$ and the final pixel size, for the binned images, was $\Delta r=0.134^{\prime \prime}$. Seeing conditions and telescope set-up limited the spatial resolution to $0.3-0.5^{\prime \prime}$ for broad-band images. The time interval between two successive images and two successive spectral series was $2.5 \mathrm{~s}$ and $75 \mathrm{~s}$ respectively.

A sub-array of approx. $30^{\prime \prime} \times 30^{\prime \prime}$ made up the final image series after the assessment for dark current, flat field, image motion, and tracking loss of the telescope. In this work we refer to 608 broadband frames that correspond to the conclusive 40-min sequence. In order to reduce the noise and take into account the instrumental profile, we applied a Wiener filter (Hunt 1984) to broadband images. We assumed a simple gaussian PSF $\left(F W H M=0.4^{\prime \prime}\right.$, comparable to the spatial resolution of the observations) and a $S / N$ ratio equal to 15 , which was found to represent a good compromise between the level of restoration and the noise amplification. 

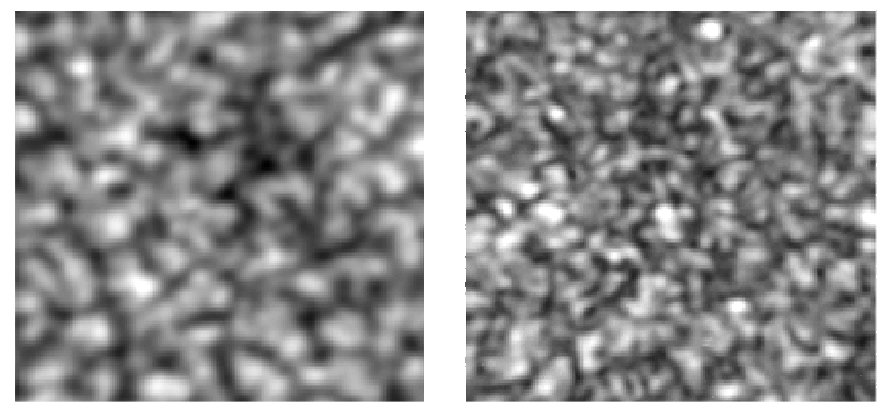

Fig. 6. The THEMIS average intensity (left panel) and rms (right panel) field as obtained by the $40 \mathrm{~min}$ time-series. The image size is $\approx 27^{\prime \prime} \times 27^{\prime \prime}$ as resulting after $k-\omega$ filtering.

The observations from the DST consist of a sequence of broadband images acquired on October 16, 1996 (from 14:30 UT to about 16:30 UT). Granulation frames were imaged through an interference filter at $550 \mathrm{~nm}$, with $10 \mathrm{~nm}$ FWHM. The images were recorded with a Videk 8-bit camera, $1360 \times 1036$ pixels, equipped with an automatic frame selection system. Within an interval of about $2 \mathrm{~s}$, the best image among 12, i.e. the one with the highest rms value, was retained and read by the camera. Each of the images was acquired with an exposure time of $8 \mathrm{~ms}$. The total interval between successive images was 4.7 s. A sub-portion of $1024 \times$ 1024 pixels made up the final images. The image scale was set at $0.123 \mathrm{arcsec} / \mathrm{pixel}\left(\Delta r=0.123^{\prime \prime}\right)$, for a total field of view of about $2 \times 2$ arcmin. Seeing conditions were excellent, and the use of the DST granulation tracker (Rimmele et al. 1991) allowed a further improvement of the image stability. For a great part of the sequence, the images are nearly diffraction limited (0.19 arcsec for the DST). After the standard dark current and flat field corrections, a destretching algorithm was applied to remove the atmospheric distortion in the white light images. More details can be found in Cauzzi et al. (1998). The final dataset covers a period of $1 \mathrm{~h} 20 \mathrm{~m}$ (512 images) with a time resolution of $9.4 \mathrm{~s}$.

As shown by e.g., Title et al. (1989), the 5-min oscillations must be removed from the intensity images before analyzing the granulation pattern. We hence applied a 3-dimensional Fourier filter in the $k-\omega$ domain that removed all Fourier components with $\omega / k>4.7 \mathrm{~km} \mathrm{~s}^{-1}$ and $\omega / k>6 \mathrm{~km} \mathrm{~s}^{-1}$, for DST and THEMIS observations, respectively.

In order to investigate the occurrence of a quasi-regular structure and of persistence in the photospheric intensity fields we have time-averaged the granulation series over the entire observing periods to recover the mean granulation pattern. In Figs. 6 and 7 we report the average intensity fields along with the corresponding rms intensity field as evaluated by the THEMIS and DST observations, respectively. As shown in several studies (Getling \& Brandt 2002; Baudin et al 1997), the resulting averaged fields are not smeared out, showing, conversely, still persistent granular-like patterns. Moreover, also the rms fields are also strongly structured, showing the occurrence of localized sites of fluctuations. We postpone the study of these features to a future work.

On single snapshots and the average images of the intensity fields the compact solar structures have been extracted
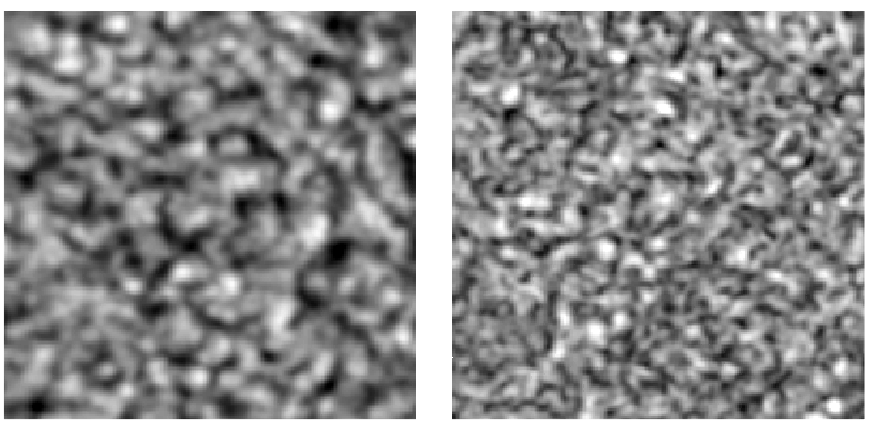

Fig. 7. The DST average intensity (left panel) and rms (right panel) field as obtained by the 90 min time-series. The image size is a portion of $\approx 27^{\prime \prime} \times 27^{\prime \prime}$ of the original image.
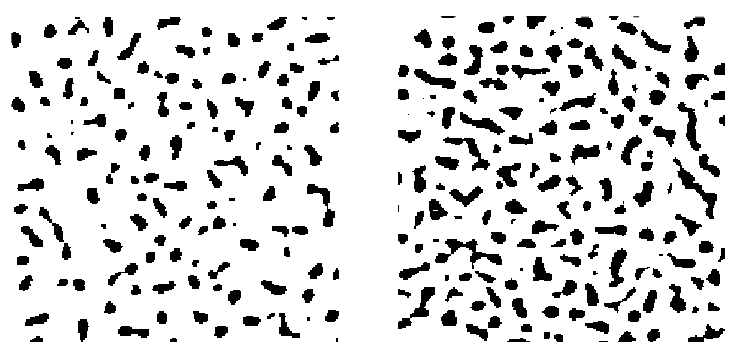

Fig. 8. The binarized images of THEMIS (left panel) and DST (right panel) average intensity field reported in Fig. 6 and 7. Images size is $27^{\prime \prime} \times 27^{\prime \prime}$ for convenience.

using a numerical binarization procedure based on a dynamical thresholding technique as described in Berrilli et al. (1998, 2002). In more detail, the dynamical threshold $I_{\text {thr }}$ depends on the pixel position and on the statistical properties of a moving box centred on the selected pixel according to the following expression:

$I_{\mathrm{thr}}=\left\langle I_{\mathrm{box}}\right\rangle+\xi \times \sigma_{\mathrm{box}}$

where the $\left\langle I_{\text {box }}\right\rangle$ and $\sigma_{\text {box }}$ are, respectively, the intensity average and the fluctuation rms in the moving box, and $\xi$ is a parameter. The choice of the moving box size $L_{\text {box }}$ and of the $\xi$ parameter has been accomplished by optimizing the area and the number of extracted structures in the $\left(L_{\mathrm{box}}, \xi\right)$ parameter space. The used optimal values are $L_{\text {box }} \simeq 2^{\prime \prime}$ and $\xi=0.7$. Although the used binarization procedure is not optimized in order to extract the correct shape and dimension of solar granular structures as in Bovelet \& Wiehr (2001), the effects on the normalised information entropy $H^{\prime}(r)$ results are negligible as discussed in Appendix A, being limited only to a slight shift of the first peak position and to the absolute value of $H^{\prime}(r)$ maxima.

In Fig. 8 we report the binarized image relative to the averaged intensity field of THEMIS and DST time series. From a comparative look at these binarized images we may note a difference in the average dimension of the extracted granular structures, as also confirmed by the area coverage of the structures which is $\sim 14 \%$ and $\sim 21 \%$ for THEMIS and DST images, respectively. We believe that a possible explanation of the observed difference would be the different time interval over which the averaging procedure is performed $(\sim 40 \mathrm{mn}$ and $\sim 1 \mathrm{~h} 20 \mathrm{mn}$ for THEMIS and DST, respectively) which tends to smear the structures. 

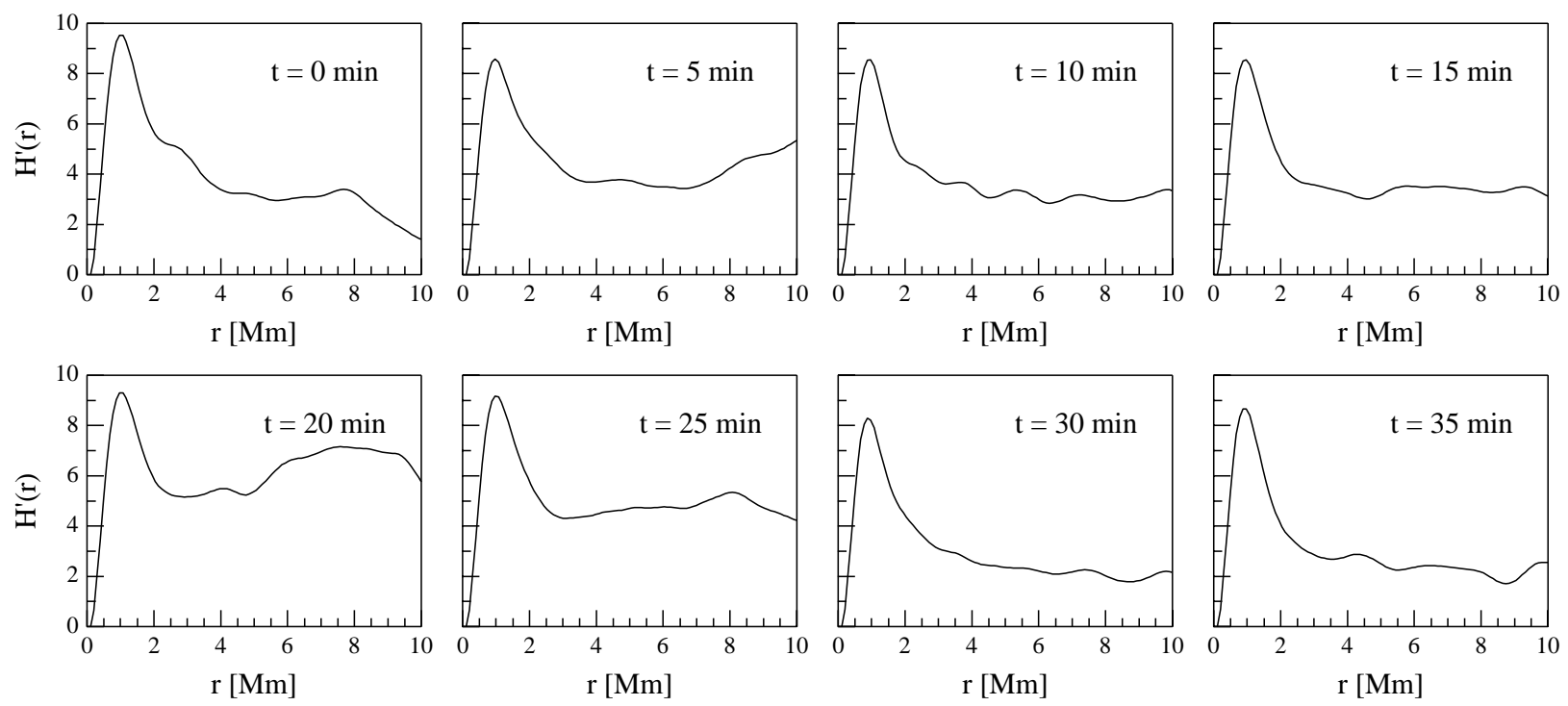

Fig. 9. The normalised information entropy $H^{\prime}$ at different times for the THEMIS binarized intensity fields.

\section{Results and discussion}

In this section we will show and discuss the results of information entropy analysis on broadband THEMIS and DST observations. We underline that for THEMIS observations in conjunction with broadband images we have also collected spectral images by which an analysis similar to the one described in this work can be applied to velocity fields at different photospheric heights. This will be the object of a second paper in preparation at the moment.

In passing, we want to remark that in order to compare the results coming from the two different observational campaigns we have limited our analysis to the range of scales less than $10 \mathrm{Mm}$. This scale which corresponds to about half of the linear size of THEMIS images, has been set in order to have a good statistics as previously discussed in Sect. 2. We postpone the investigation of larger scales and the topology at these scales to a future work.

The first step of our approach to the possible existence of a topological ordering in the granulation field is to investigate the behaviour of the normalised information entropy measure in single snapshots of the granulation field. In order to do this we have selected a subset of 32 images with the highest image quality among THEMIS observation. Each image is the best one in a subset of 9 images. The subsets have been chosen to be timely separated by $\sim 1 \min 15 \mathrm{~s}$.

In Fig. 9 we report the normalised information entropy $H^{\prime}(r)$ for a set of THEMIS binarized intensity fields at different times. In all the plots the behaviour of $H^{\prime}(r)$ as a function of the box-scale $r$ is characterized by a pronounced peak located at the scale $r \sim 1 \mathrm{Mm}$. At larger scales $H^{\prime}(r)$ displays a non-monotonous trend with the box-dimension $r$, showing secondary maxima that evolve with time. As stated by Van Siclen (1997), $H^{\prime}(r)$ values larger than zero are related with clustering effects. Thus, the location of the first peak of $H^{\prime}(r)$ must be read as the consequence of the clustering effect of black pixels related to the granules. In other words, this characteristic scale of $\sim 1 \mathrm{Mm}$ represents an indirect measure of the granule typical size. Furthermore, the presence of secondary maxima is the evidence of clustering effects of granular structures, i.e. of clusters of granules. Similar results are recovered in the case of DST observations (data not shown here).

In order to get information on the possible existence of an average topological ordering (i.e. the existence of an average temporal-spatial correlation of the granule positions) we have applied to the $H^{\prime}(r)$ plots relative to all the selected set of images, the approach described in Sect. 2 to decompose $H^{\prime}(r)$ in a superposition of contributions. The decomposition is accomplished by a nonlinear best fit procedure (LevembergMarquardt algorithm) of the $H^{\prime}(r)$ using Eq. (7) with $k=5$. The optimal number $n$ of contribution functions was determined by analysing the trend of the reduced $\chi_{\mathrm{r}}^{2}(n)$ as a function of the number of contributions. Figure 10 shows the behaviour of the quantity $\chi_{\mathrm{r}}^{2}(n)$. Larger numbers of contributions do not, indeed, reduce $\chi^{2}(n)$. Figure 11 shows an example of such a decomposition of $H^{\prime}(r)$ using Eq. (7) for two snapshots of THEMIS and DST observations. This decomposition has been applied to all the 32 selected images and to a subset of DST observations, consisting of 64 images timely separated by an interval of $\Delta t=1 \mathrm{mn} 25 \mathrm{~s}$. In Fig. 12 it is shown the behaviour of the peak positions as a function of time in the case of THEMIS observations (upper panel) and DST ones (lower panel).

While the position of the first peak is constant with time, the positions of the other peaks fluctuate with time. First of all, we remark that these temporal-fluctuations do not show any periodicity and do not correlate with the quality images as described by the contrast. Moreover, we can identify some time intervals when the secondary aggregations of the structures are modified in the direction of an expansion of the overall arrangement of the granules (see for example the time interval from $12 \mathrm{mn}$ to $20 \mathrm{mn}$ in THEMIS observations). Due to the time duration of this effect we exclude an atmospheric origin and believe that this is due to the dynamics of the granulation fields. We name this behaviour dynamic topological deformation, meaning the occurrence of some plastic 


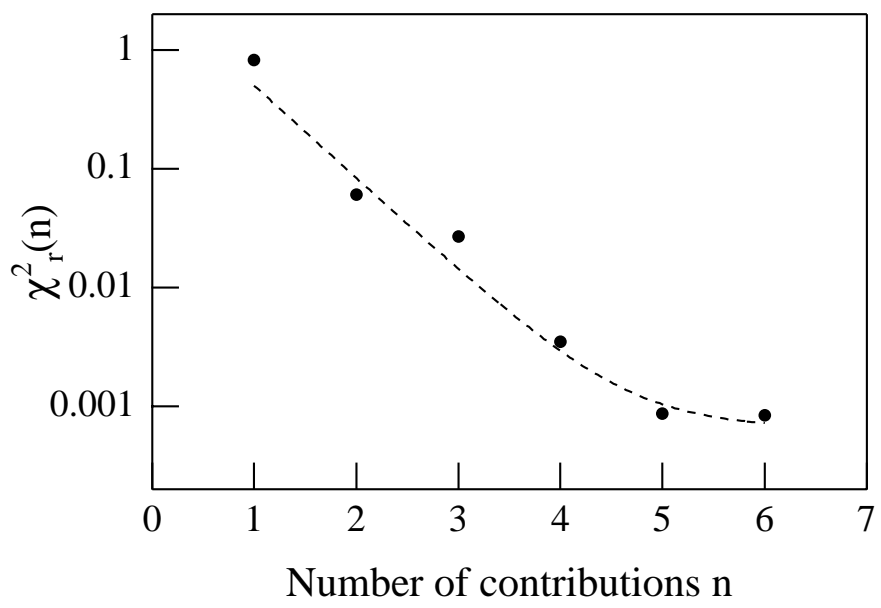

Fig. 10. The behaviour of the reduced $\chi_{\mathrm{r}}^{2}(n)$ versus the number $n$ of contributions in the case of THEMIS $H^{\prime}(r)$ decomposition. The dashed line is an eye guide.
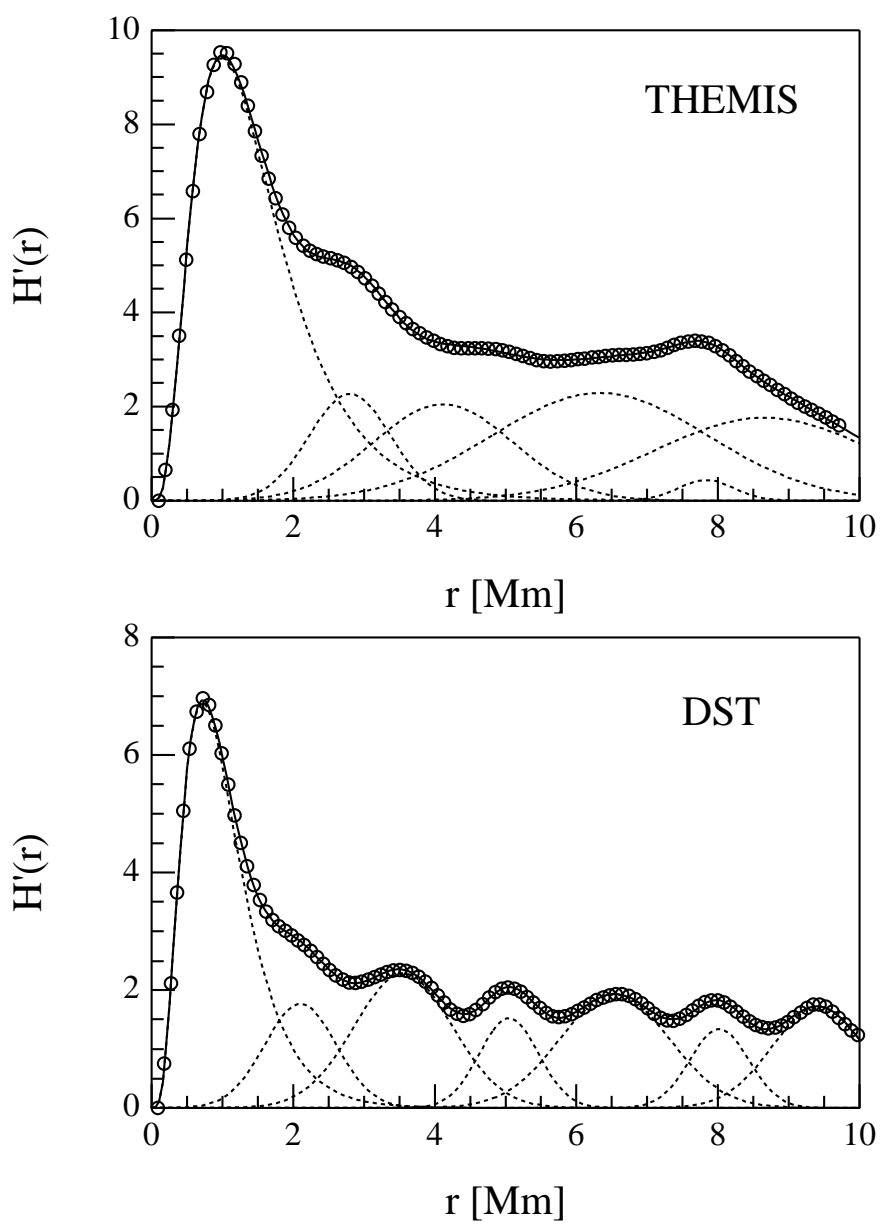

Fig. 11. The decomposition of the normalised information entropy $H^{\prime}$ for two images in the case of THEMIS (upper panel) and DST (lower panel), respectively. Solid line is the best fit using Eq. (7). The dashed lines show the single contributions.

deformations of the overall topology. As a matter of fact, if the position of secondary maxima would be fixed in time, we should think about the presence of a sort of rigid topology that would not change the shape of $H^{\prime}(r)$. Conversely, this result
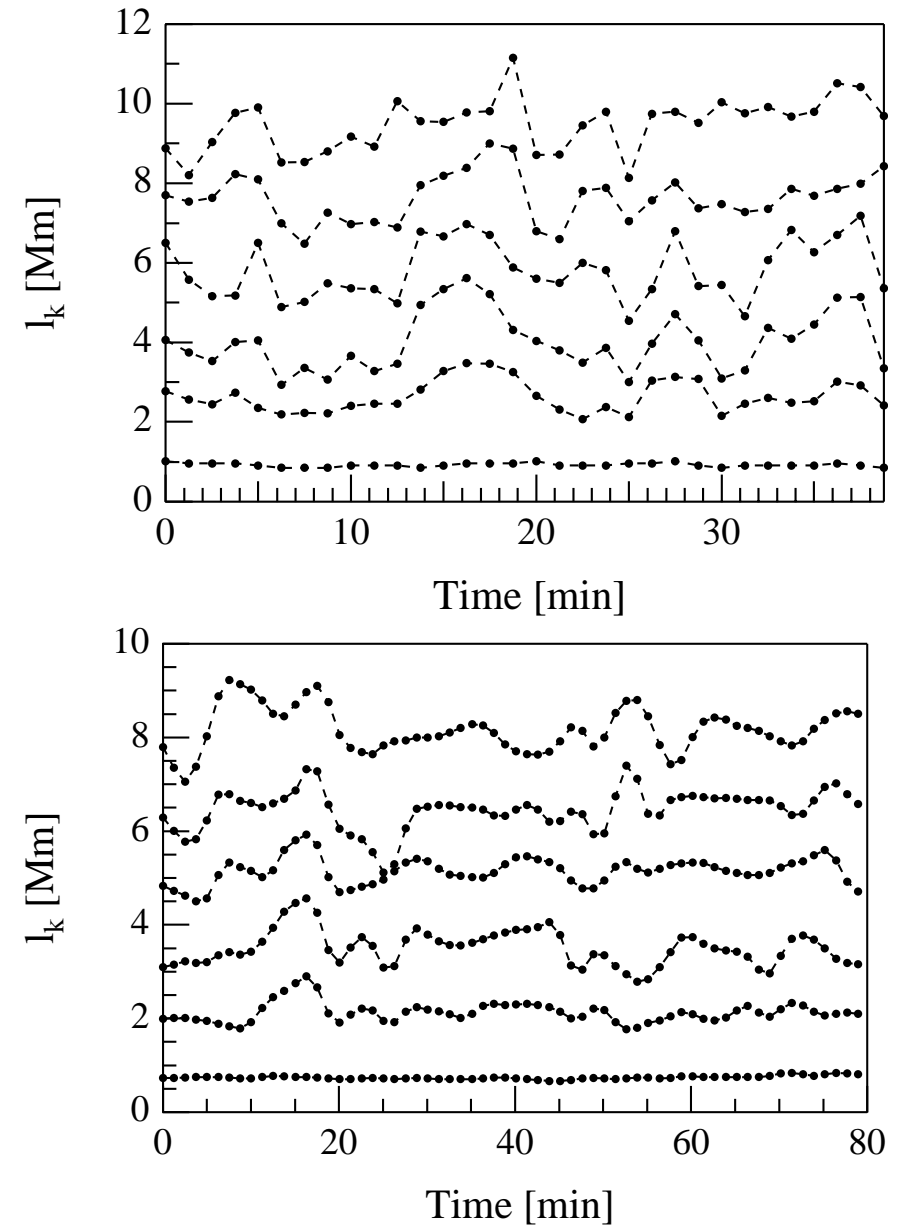

Fig. 12. The behaviour of the position of the contributions of the $H^{\prime}(r)$ decomposition for THEMIS (upper panel) and DST (lower panel), respectively.

suggests that we are in the case of a deformable topology similar to that of a fluid/amorphous system.

Starting from these results on single images we have evaluated the average positions of the secondary maxima. Averaging procedure was performed over time by considering only those values of the peak positions that fall inside the range $l_{k}^{\text {mean }} \pm 2 \sigma$, where $l_{k}^{\text {mean }}$ is the mean value evaluated on all the data set and $\sigma$ is the standard deviation. In Fig. 13 we show the average position $\left\langle l_{k}\right\rangle$ of the secondary peaks as a function of the odd numbers. A linear relationship is found suggesting the existence of a characteristic scale $l_{0}$ that is the signature of a topological ordering in the mutual positions of the granular structures. When the dependence of $\left\langle l_{k}\right\rangle$ on the odd numbers is fitted using Eq. (8) we found $l_{0}=[1.69 \pm 0.07] \mathrm{Mm}$ and $l_{0}=[1.44 \pm 0.06] \mathrm{Mm}$ for THEMIS and DST, respectively. Let us say some words on the meaning of this scale. Although this topological ordering scale $l_{0}$ could be related to the average dimension of the convective cells (see the discussion in the next section), this characteristic scale plays the same role of the lattice constant, being related with the presence of a quasi-regular pattern in the intensity field. We remark that, to our knowledge, this result is the first quantitative evidence of a spatial ordering in the granulation pattern. In passing we anticipate that similar results have 


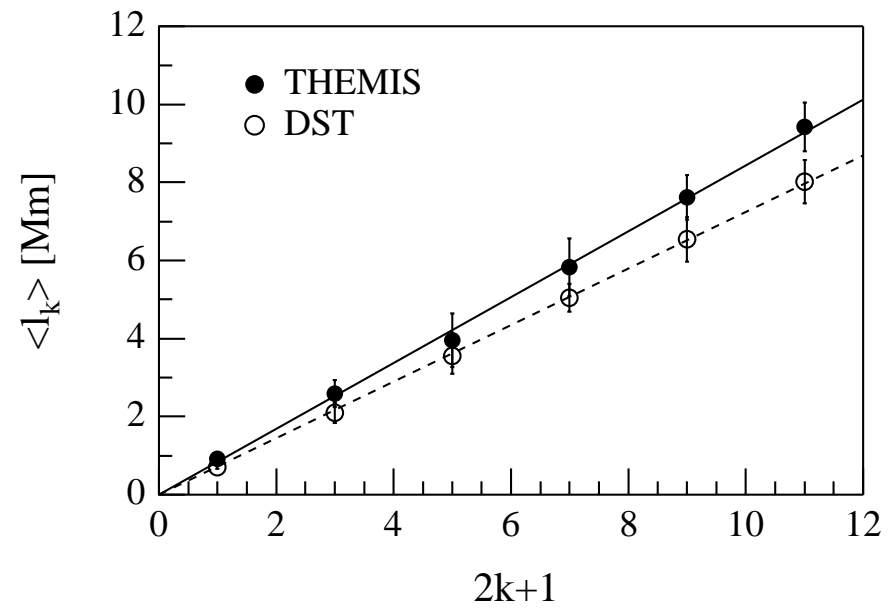

Fig. 13. The average positions of the peaks of the $H^{\prime}(r)$ decompositions on single images for THEMIS (black circles) and DST (empty circles) observations. Error bar represents the standard deviation. The solid and dashed lines are linear best fits using the expression Eq. (8) of Sect. 2.

been found analysing velocity fields as observed in C I line at $\lambda=538.0 \mathrm{~nm}$, which is representative of the deeper layers in the solar photosphere.

Another relevant aspect that can be investigated is the persistence of such a spatial topological ordering. As a matter of fact, looking to the granular pattern evolution with time we can immediately realize that granules tend to be born in the nearby sites of pre-existing granular structure. In order to visualize this aspect of the granulation pattern evolution we have constructed an image as the sum of DST images binarized following the procedure described in Sect. 3. Figure 14 shows a 3-d view of the resulting image (upper panel) along with a 2-d view (lower panel). The existence of a multipeaked structure of the resulting pattern from this image is a clear evidence of persistence in the granulation intensity field. We emphasize two aspects related to this persistence in binarized images: $i$ ) the persistent granularlike patterns reported in Figs. 6 and 7 are not due to particular bright intensity structures; $i$ ) we found more than 30 local regions (zero points in the image) that are never sites of a granule.

Figure 15 shows the evolution of the normalised information entropy $H^{\prime}(r)$ as evaluated by averaging the DST images with time. These plots clearly shows a temporal evolution of the secondary $H^{\prime}(r)$ maxima. The observed evolution could be related to the temporal emergence of structures and/or pattern at scales usually associated with mesogranulation. Since its discovery, the spatial and temporal characteristics related to mesogranulation have been difficult to determine accurately (Rieutord et al. 2000). Anyway, the typical spatial and temporal scales associated with mesogranulation range within length scale between $3 \mathrm{Mm}$ and $10 \mathrm{Mm}$ and time scales between $30 \mathrm{~min}$ and $6 \mathrm{~h}$. In order to get some insight on mesogranulation from the normalised information entropy measure analysis we have investigated the change of the $H^{\prime}(r)$ with averaging time at three different range of scales reported in literature: 3.5-4.5 Mm (Lawrence et al. 2001), 4.0-7.0 Mm (Shine et al. 2000), and 5.0-10.0 Mm (November et al. 1981).
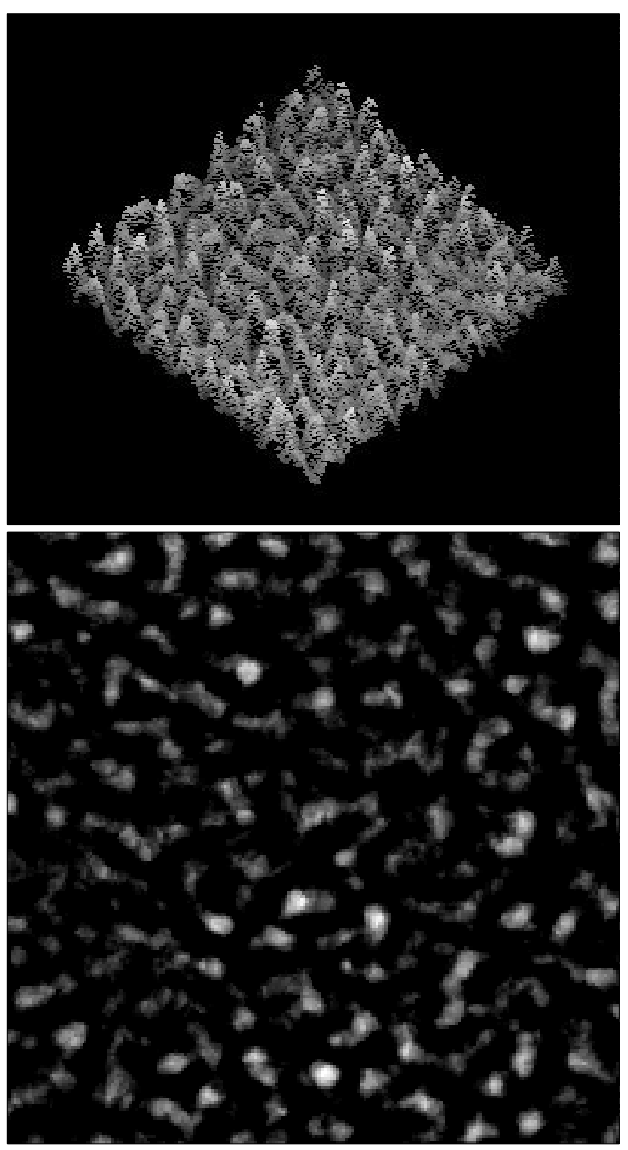

Fig. 14. The persistency pattern for the granulation field as evaluated from DST binarized images. The upper panel shows a 3-d view of this pattern where the multipeaked structure is clearly evident. The lower panel shows a 2-d view of the same persistence pattern where only the part of the signal that exceeds the RMS value is shown.

In Fig. 16 we report the evolution of the $H^{\prime}(r)$ percentage contribution $I_{H}(\Delta r)$ with averaging time $\Delta t$ in the three above mentioned ranges of scales, as defined according to the following expression:

$I_{H}(\Delta r)=\frac{\int_{r_{1}}^{r_{2}} H^{\prime}(r) \mathrm{d} r}{\int_{0}^{r_{\mathrm{M}}} H^{\prime}(r) \mathrm{d} r}$

where $r_{1}$ and $r_{2}$ are the extremes of the range of scales, and $r_{\mathrm{M}}$ is the maximum length scale here investigated $\left(r_{M}=10 \mathrm{Mm}\right)$. This figure allows us to trace in time the statistical weight of the three selected ranges of scales. In detail, the $I_{H}(\Delta r)$ at 5.0-10.0 Mm shows a clear evolution with averaging time, the information entropy percentage contribution at the other two ranges of scales is nearly constant. This result suggests the existence of a spatial coherence with a characteristic time scale of $\sim 40 \div 60 \mathrm{~min}$ at scales larger than $5.0 \mathrm{Mm}$.

Figure 17 shows the $H^{\prime}(r)$ along with its decomposition for the DST binarized average intensity field. Similar results have been found in the case of THEMIS average intensity field (data not shown). We have decomposed the $H^{\prime}(r)$ in a superposition of contributions following the same procedure applied for single images. Figure 18 reports the positions $l_{k}$ of the contribution peaks vs. odd numbers for both DST and THEMIS 

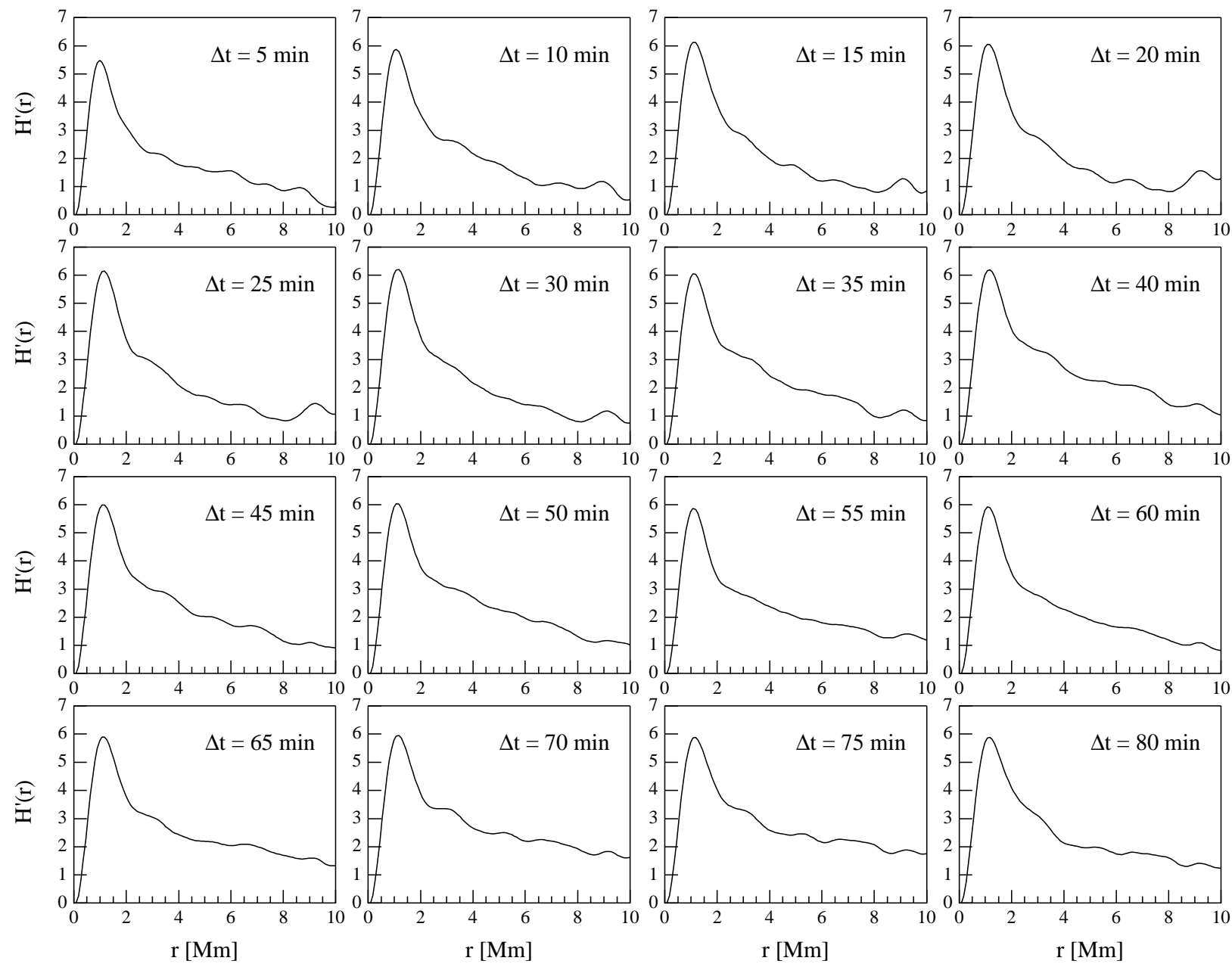

Fig. 15. The evolution of the normalised information entropy $H^{\prime}$ by averaging DST images with time.

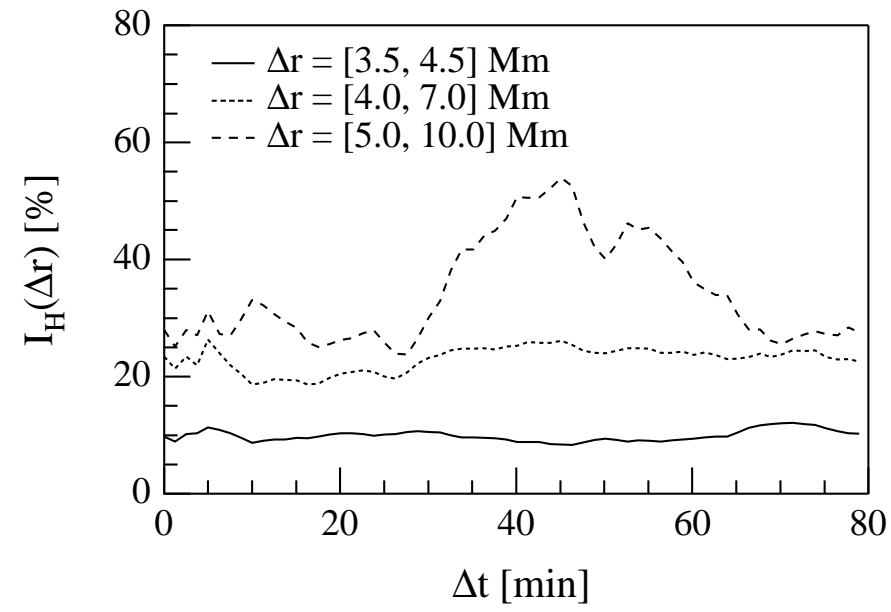

Fig. 16. Percetange contribution to the overall information entropy $H^{\prime}(r)$ in three different range of scales $\Delta r$ as a function of averaging time $\Delta t$.

results. A linear relationship is recovered in both cases, and using Eq. (8) we can define two characteristic length scales: $l_{0}=[1.55 \pm 0.03] \mathrm{Mm}$ and $l_{0}=[1.53 \pm 0.05] \mathrm{Mm}$ for DST and THEMIS, respectively. These values do not meaningfully differ from the topological ordering scale found on single images,

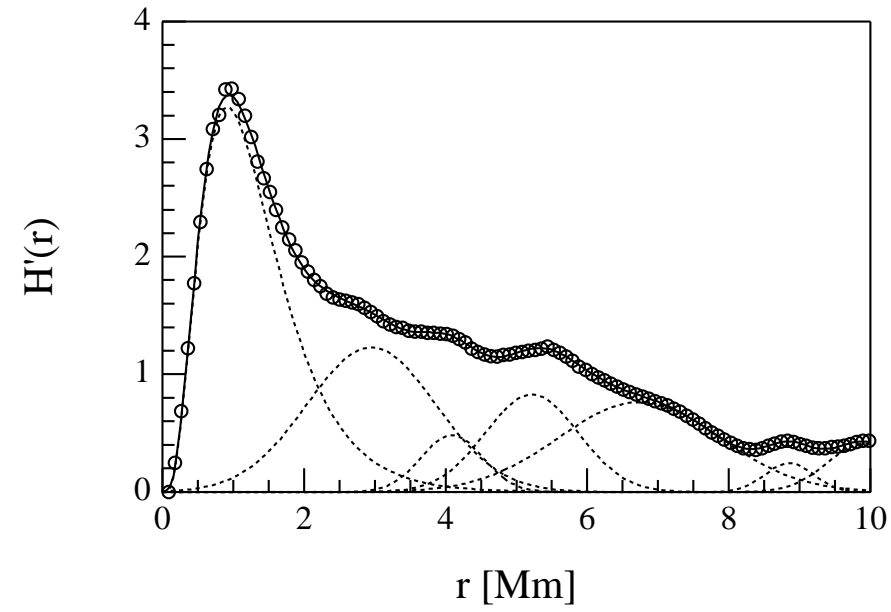

Fig. 17. The normalised information entropy $H^{\prime}$ (empty circles) for the DST binarized average intensity field. Solid line is the best fit using Eq. (7) with $k=6$. The dotted lines show the single contributions. Only contributions with maxima inside $10 \mathrm{Mm}$ are shown for convenience.

suggesting that topological ordering is an intrinsic feature of the convective pattern as observed by means of intensity fields. 


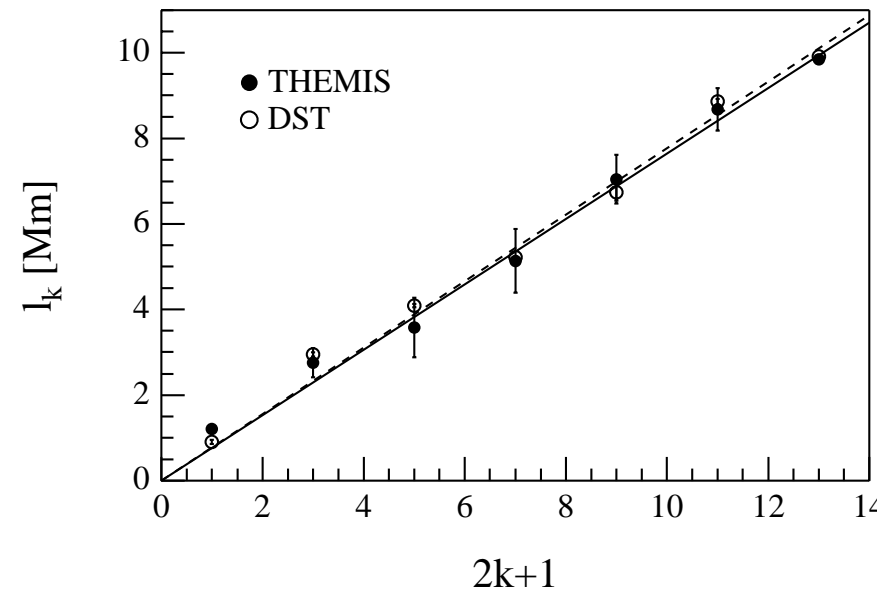

Fig. 18. The peak positions of the $H^{\prime}(r)$ contributions versus odd numbers for THEMIS (solid circles) and DST (empty circles) average intensity fileds. The solid and dashed lines are linear fit for THEMIS and DST, respectively.

\section{Summary and conclusions}

In this work, we have tackled the highly debated topic of regular structures in the solar photosphere (Getling \& Brandt 2002; Rast 2002) by means of information theory. In detail using the normalised information entropy measure, as defined by Van Siclen (1997), we have quantitatively investigated the existence of a hidden ordering in the topology of the granular structures. Our findings may be summarized as follows:

1. The normalised information entropy measure $H^{\prime}(r)$ analysis shows a pronounced peak at $\sim 1 \mathrm{Mm}$ both in average and single images. This peak is related to the average length scale of the granular structures, as obtained by the numerical binarization procedure, and voids among granules.

2. Other relevant scales emerge in the $H^{\prime}(r)$ as secondary maxima. These scales are the signature of secondary clustering effects due to a non-random distribution of the granular structures.

3. The decomposition of $H^{\prime}(r)$ reveals the existence of a hidden ordering topology in both single and average granular patterns which allows us to define an average topological ordering length scale $l_{0}=[1.55 \pm 0.10] \mathrm{Mm}$. While the $\sim 1 \mathrm{Mm}$ scale shows the primary contribution due to granules, the $\sim 1.55 \mathrm{Mm}$ scale contains positional information of individual cells that recognise their position with respect to their partners. As a matter of fact, the scale we found characterizes the system as ordered and defines a correlation between parts of the system (i.e. photospheric flows) as it occurs in the case of a crystal lattice.

4. The percentage contribution $I_{H}(\Delta r)$ to $H^{\prime}(r)$ in the range $5.0 \div 10.0 \mathrm{Mm}$ shows an evolution with averaging timeinterval. This result suggests the existence of a spatial coherence with a characteristic time scale of $\sim 40 \div 60 \mathrm{~min}$ on typical scales associated with mesogranulation.

The picture that emerges from our analysis implies the presence of space-time ordering in the topology of the solar convection pattern. The space ordering, present in both single and average images, suggests the occurrence of time persistence in the origin points for granules. We remark that the observed persistence is also confirmed by the averaging procedure of the binarized images that eliminate the effects of particularly bright structures. Moreover, conversely to what stated by Getling \& Brandt (2002) the observed regularity on the granulation pattern is even revealed in single images. In other words, also the single images reveal the presence of a ordered topology, while the time average images point out the spatial persistence of this ordered topology.

Concerning the emergence of this topological ordering, we believe that the existence of a quasi-regular pattern might be understood in the framework of a complex system of space-time coupled structures with a characteristic size. Let us consider two different classes of random phenomena (Pagels 1985): $i$ ) the rain falling on a sand beach, ii) the nesting of a seabird colony on a cliff.

In the first phenomenon no regular pattern is observed either instantaneously or with increasing time due to the lack of a space-time coupling among the falling raindrops and to the total random nature of the process. This leads to a flat pattern without spatial ordering and time persistence.

Conversely, looking at the nests of a seabird colony we may recognize a sort of ordering and persistence in the nesting pattern. In this case, the process requires certain constraints on the minimum distance between two nests (the pecking distance), which is equivalent to the existence of a spatial correlation length. The emergence of a structured pattern in the nesting phenomenon is the signature of complexity and selforganization in such a random system consisting of interacting elementary subsystems.

In the case of solar granulation pattern, we retain that this complex system may be considered to some extent equivalent to the nesting process, where the role of the average distance between two nests is played by typical correlation scale between convective matter flows. Here, the self-organization, which comes out from the inherent spatial symmetry breaking due to the convective process, induces a spatial ordering. Our analysis reveals the existence of such a spatial ordering characterized by a length scale $l_{0} \sim 1.6 \mathrm{Mm}$. The emerging picture looks similar to our previous findings on the scaling features of the velocity fields (Consolini et al. 1999a, 1999b).

In conclusion, our analysis on the topology of the granulation pattern, as extracted using the binarization procedure on intensity photospheric fields, suggests the existence of a certain amount of regularity in the solar convection pattern. However, a definitive answer on the existence of a regular convective pattern requires the investigation of velocity fields that better describe the matter flow topology. We recall that intensity field, being related to the temperature field (i.e. to the advected scalar quantity), might give a non-exhaustive view of the underlying convective field. A detailed analysis of the velocity field pattern topology will be presented in a forthcoming paper.

Acknowledgements. We wish to thank the THEMIS staff for the efficient support in the observations and in particular we are grateful to C. Briand, G. Ceppatelli and G. Mainella. The authors are indebted to NSO/SP-DST staff for the telescope time allocation and the invaluable 

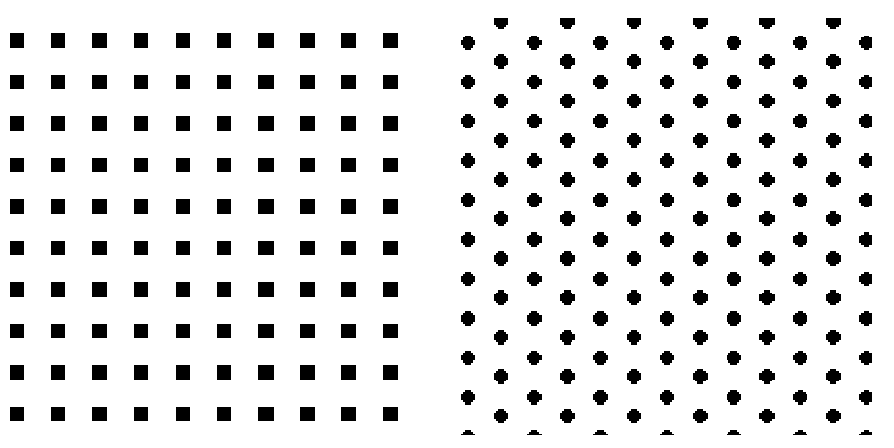

Fig. A.1. Two periodic patterns of structures: a square lattice (left panel) and a triangular lattice (right panel).

help during the observations. We would like also to thank T. Straus and G. Cauzzi for providing DST images and k- $\omega$ filtering. Special thanks to P.N. Brandt and M.P. Rast for useful discussions. This work was partially supported by the Italian National Research Council (CNR) grant Agenzia2000 CNRC0084C4.

This work is partially based on observations made with THEMISCNRS/INSU-CNR operated on the island of Tenerife by THEMIS S.L. in the Spanish Observatorio del Teide of the Instituto de Astrofisica de Canarias.

\section{Appendix A}

In this Appendix we present the results of information entropy analysis on some meaningful simulated periodic and non-periodic patterns. All the patterns here considered are 200 pixels $\times 200$ pixels two-level images. In all these cases the evaluation of information entropy measure has been limited to the range $1 \leq m \leq 100$ for statistical meaning.

In Fig. A.1 we report two periodic configurations: a square periodic pattern with a lattice length $m_{\mathrm{r}}=20$ pixels (left panel), and a triangular periodic pattern with a lattice length $m_{\mathrm{r}}=19$ pixels (right panel). In passing we note that the triangular periodic pattern derives from a hexagonal regular tessellation of the plane where the structures lie in the centre of hexagons. The behaviour of $H^{\prime}(m)$ for the two patterns is shown in Fig. A.2. It is evident how the normalised information entropy $H^{\prime}(m)$ is able to capture the periodicity of the lattice. In detail, as argued by Van Siclen (1997) while the minima reveal the underlying periodicity, as sampled by the squared $m \times m$ sliding box, the maxima are also related with the typical length scale of the regions devoid of black pixels. Indeed, in this analysis a region devoid of black pixels must also be considered as a structure giving a nonvanishing contribution to the information entropy.

In the following we report the results on different types of random patterns in order to clarify how randomness affects the information entropy measure analysis. In detail, we investigate the case of a random distribution of structures on a regular pattern, and of a total randomness. We remark that in order to compare the results coming from these different patterns we have also fixed the number of structures in each pattern to be exactly 100 .

Figure A.3 shows two different pseudorandom distributions on regular patterns. In the first case (left panel) we have
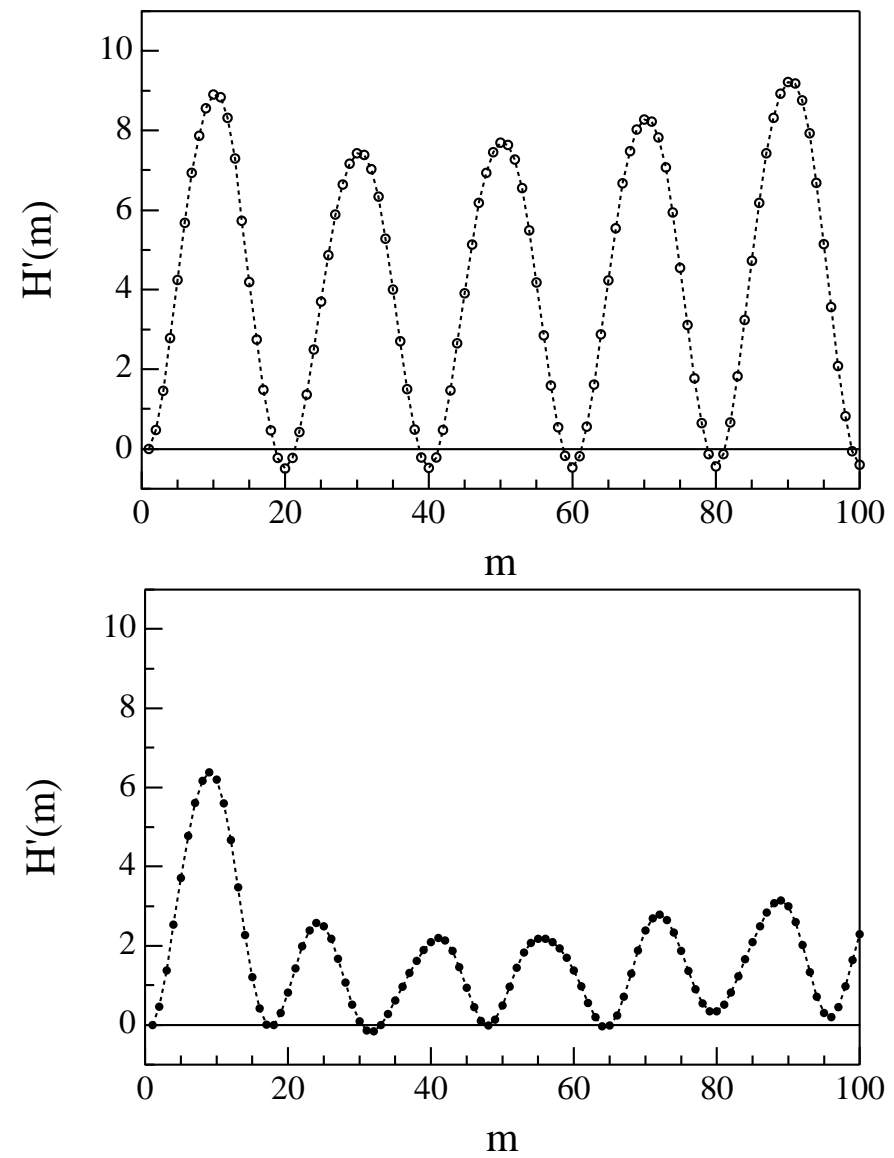

Fig. A.2. The normalised information entropy $H^{\prime}(m)$ of the two patterns reported in Fig. A.1. Upper and lower panel refer to the square and triangular lattice, respectively.
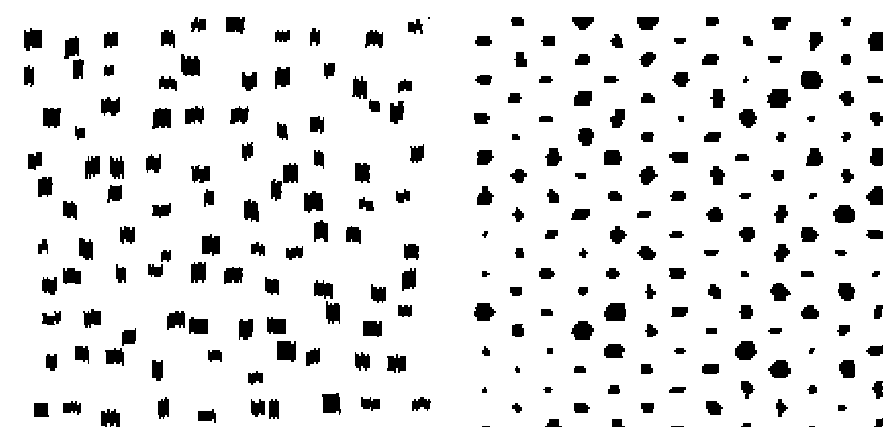

Fig. A.3. Two periodic patterns of structures: a square lattice (left panel) and a triangular lattice (right panel).

a regular triangular pattern with structures of random shapes located on the lattice sites. The second case refers to the same pseudorandom pattern shown in Fig. 3, but where the structures have random shapes. The lattice constants of the two hidden regular patterns are $m_{\mathrm{r}}=19$ pixels and $m_{\mathrm{r}}=20$ pixels for the triangular and square patterns, respectively. The results of the analysis of the normalised information entropy measure on the two patterns are reported in Fig. A.4. We note that the different degree of randomness of the two patterns reflects on the different behaviour of $H^{\prime}(m)$ : a higher randomness corresponds to a less evidence of the secondary maxima. 

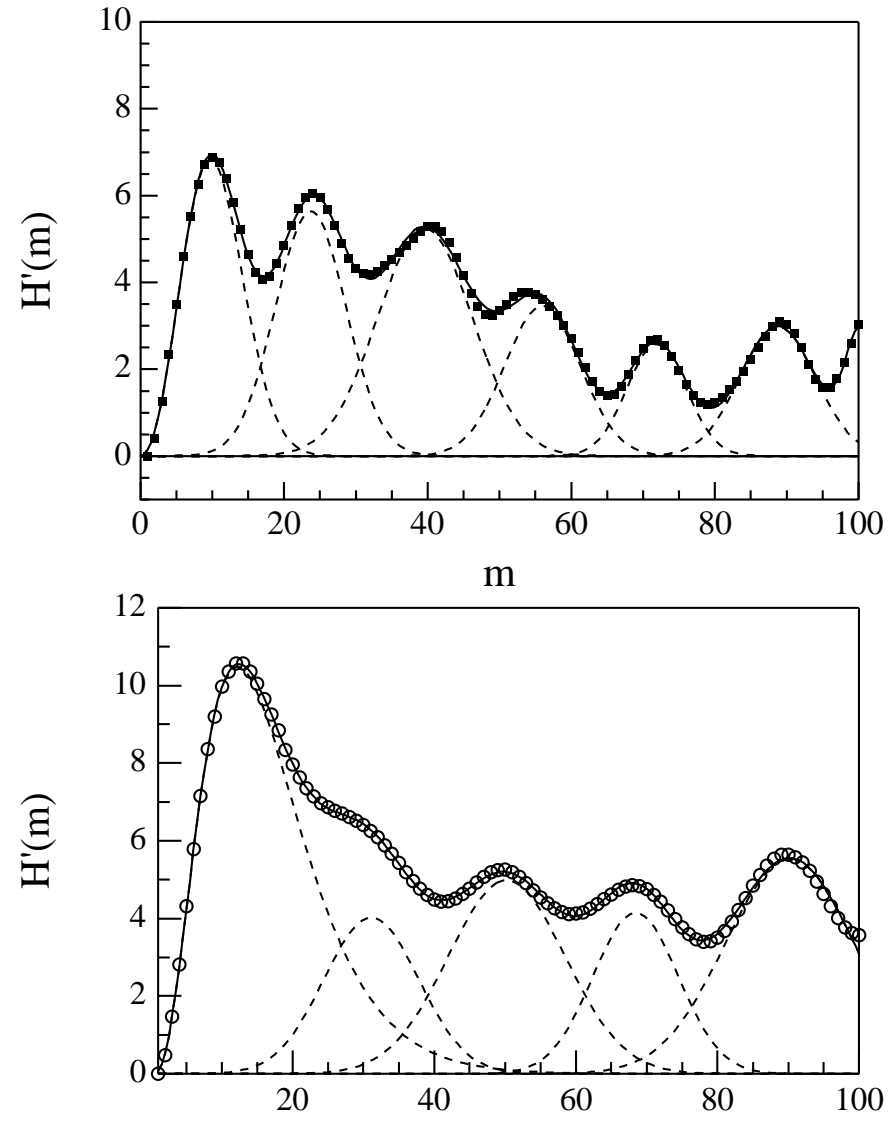

$\mathrm{m}$

Fig. A.4. The $H^{\prime}(m)$ behaviour for the two random patterns reported in Fig. A.3. Upper and lower panels refer to triangular and square lattice. Solid line is the best fit of $H^{\prime}$ using Eq. (7). Dashed lines are the single contributions.

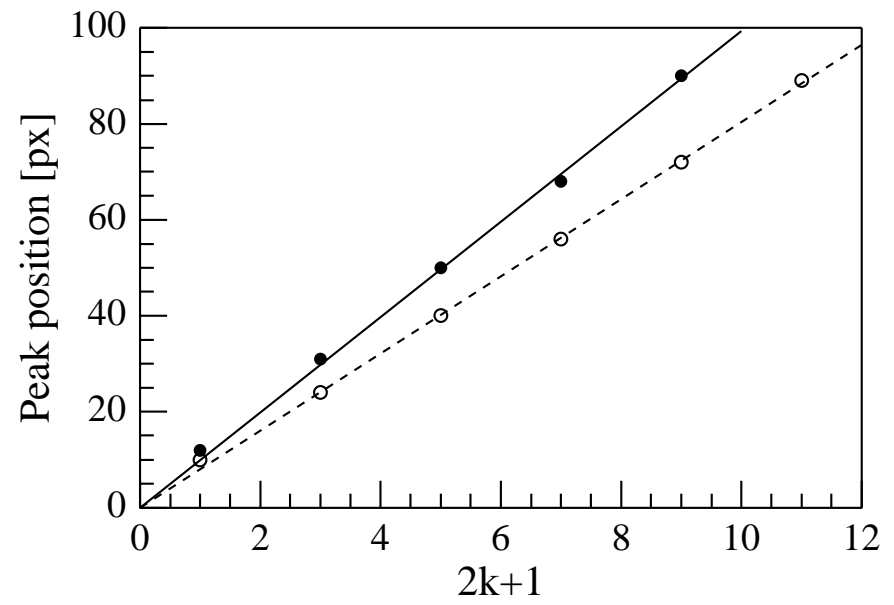

Fig. A.5. The trends of the peak positions versus the odd numbers for the two decompositions shown in Fig. A.4. Empty and solid circles indicate the peak positions for the triangular and square lattice, respectively. Solid and dashed lines are linear best fits.

In applying the same procedure discussed in Sect. $2, H^{\prime}(m)$ for the above random patterns has been decomposed in a superposition of a set of contribution functions. The decompositions are also shown in Fig. A.4. The linear relationship

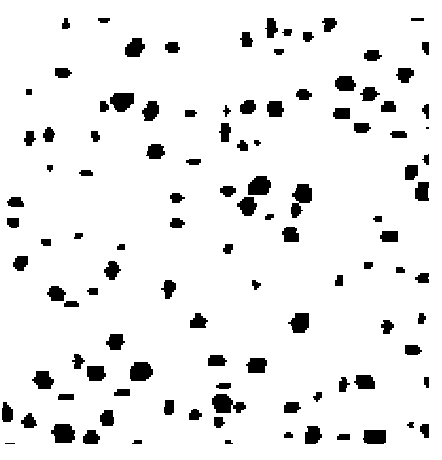

Fig. A.6. A snapshot of a pseudorandom pattern of non-overlapping structures.

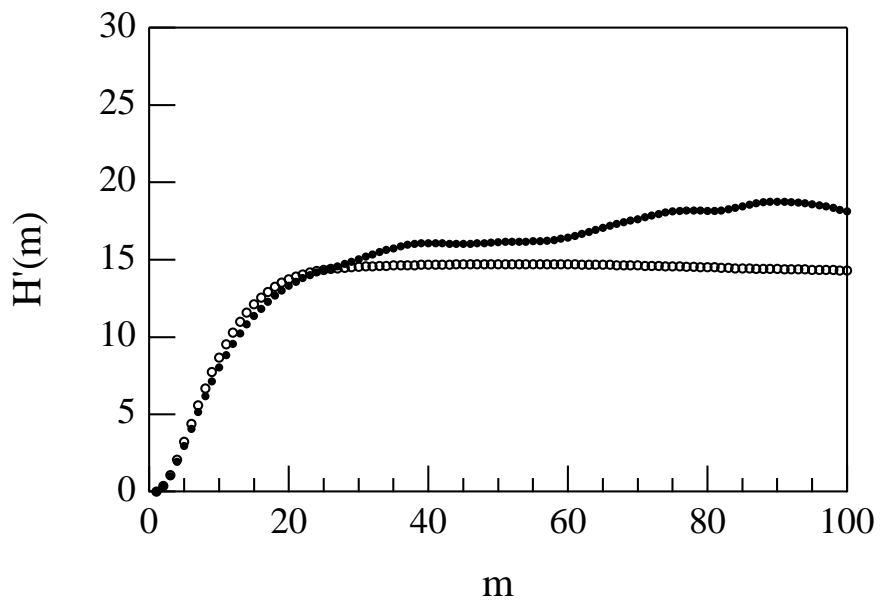

Fig. A.7. $H^{\prime}(m)$ for the single snapshot reported in Fig. A.6 (solid circles) and the set of 1000 pseudorandom patterns (empty circles).

between the peak positions of the contributions and the odd numbers (Fig. A.5) allows us to extract the underlined periodicity of the patterns and to derive the lattice characteristic lengths: $m_{\mathrm{r}}=16$ pixels (triangular lattice) and $m_{\mathrm{r}}=20$ pixels (square lattice). The extracted $m_{\mathrm{r}}=16$ pixels characteristic length for the triangular lattice exactly corresponds to the lattice constant of $m_{\mathrm{r}}=19$ pixels when the triangular pattern is investigated using a square moving box. As a matter of fact the extracted value results from the projection of the lattice vector on the orthogonal reference system of the moving box.

Finally, we have studied the behaviour of $H^{\prime}(m)$ in the case of a pseudorandom pattern consisting of a set of random shapes pseudorandomly distributed (see Fig. A.6). The only constraint in the construction of this image is the requirement that the structures do not overlap each other. That limits the maximum size of the structures. Moreover, we have also studied the $H^{\prime}(m)$ averaged on a set of 1000 pseudorandom patterns generated as described above. Figure A.7 shows $H^{\prime}(m)$ for a single snapshot and for a set of 1000 pseudorandom patterns. Both single and average $H^{\prime}(m)$ monotonously increase to the average scale of the empty zone that is about 19-20 pixels. We remember that information is also contained in the empty zones. Moreover above this scale $H^{\prime}(m)$ does not display any evident secondary maxima as a consequence of the absence of any regularities, showing a totally flat trend for the average information entropy. The flat behaviour at larger scales reflects the non-increasing 
content of information at scales larger than the ones relative to the structures.

\section{References}

Andraud, C., Beghdadi, A., \& Lafait, J. 1994, Physica A, 207, 208

Andraud, C., Beghdadi, A., Haslund, E., et al. 1997, Physica A, 235, 307

Baudin, F., Molowny-Horas, R., \& Koutchmy, S. 1997, A\&A, 326, 842

Beck, C., \& Schlögl, F. 1993, Thermodynamics of chaotic systems (Cambridge, UK: Cambridge University Press)

Berrilli, F., Caccin, B., Cantarano S., et al. 1997, Il Nuovo Cimento, 20, 297

Berrilli, F., Florio, A., \& Ermolli, I. 1998, Sol. Phys., 180, 29

Berrilli, F., Consolini, G., Pietropaolo, E., et al. 2002, A\&A, 381, 253

Bovelet, B., \& Wiehr, E. 2001, Sol. Phys., 201, 13

Cattaneo, F., Lenz, D., \& Weiss, N. 2001, ApJ, 563, L91

Cauzzi, G., Consolini, G., Berrilli, F., et al. 1998, Memorie SAIt, 69, 647

Cavallini, F. 1998, A\&A, 128, 589

Consolini, G., Carbone, V., Berrilli, F., et al. 1999a, A\&A, 344, L33

Consolini, G., Berrilli, F., Pietropaolo, E., et al. 1999b, in Magnetic Fields and Solar Processes, ed. A. Wilson, ESA SP-488, 209

Frisch, U. 1995, Turbulence - The Legacy of A.N. Kolmogorov (Cambridge: Cambridge University Press)

Getling, A. V., \& Brandt, P. N. 2002, A\&A, 382, L5

Hoekzema, N. M., Brandt, P. N., \& Rutten, R. J. 1998, A\&A, 333, 322

Hunt, B. R. 1984, Image Restoration in Digital Image Processing Techniques (Academic Press, Inc.)

Lawrence, J. K., Cadavid, A. C., \& Ruzmaikin, A. A. 1999, ApJ, 513, 506

Lawrence, J. K., Cadavid, A. C., \& Ruzmaikin, A. A. 2001, Sol. Phys., 202, 27
Legrenzi, P. 2002, Prima lezione di scienze cognitive (Editori Laterza, Bari)

Müller, D. A. N., Steiner, O., Schlichenmaier, R., et al. 2001, Sol. Phys., 203, 211

Nordlund, Å., Spruit, H. C., Ludwig, H.-G., et al. 1997, A\&A, 328, 229

November, L. J., Toomre, J., Gebbie, K. B., et al. 1981, ApJ, 245, L123

Pagels, H. R. 1985, Perfect Symmetry - The Search of the Beginning of Time (New York: Bantam Books)

Ploner, S. R. O., Solanki, S. K., \& Gadun, A. S. 2000, A\&A, 356, 1050

Rast, M. P. 1999, in High Resolution Solar Physics: Theory, Observations and Techniques, ed. T. R. Rimmele, K. S. Balasubramaniam, \& R. R. Radick, p. 443

Rast, M. P. 2002, A\&A, 392, L13

Rieutord, M., Roudier, T., Malherbe, et al. 2000, A\&A, 357, 1063

Rimmele, Th., von der Luehe, O., Wiborg, P. H., et al. 1991, SPIE, 1542,186

Roudier, Th., Malherbe, J. M., November, L., et al. 1997, A\&A, 320, 605

Shannon, C. E., \& Weaver, W. 1959, The mathematical theory of communication (Urbana: University of Illinois)

Shine, R. A., Simon, G. W., \& Hurlburt, N. E. 2000, Sol. Phys., 193, 313

Stein, R. F., \& Nordlund, Å. 1998, ApJ, 499, 914

Spruit, H. C., Nordlund, Å., \& Title, A. M. 1990, ARA\&A, 28, 263

Starck, J.-L., Murtagh, F., \& Bijaoui, A. 1998, Image Processing and Data Analysis. The Multiscale Approach (Cambridge: Cambridge University Press)

Title, A. M., Tarbell, T. D., Topka, S. H., et al. 1989, ApJ, 336, 475

Van Siclen, C. Dew. 1997, Phys. Rev. E, 56, 5211 